\title{
Weighted Committee Games
}

\author{
Sascha Kurz \\ Dept. of Mathematics, University of Bayreuth, 95440 Bayreuth, Germany \\ email: sascha.kurz@uni-bayreuth.de \\ Alexander Mayer \\ Dept. of Economics, University of Bayreuth, 95440 Bayreuth, Germany \\ email: alexander.mayer@uni-bayreuth.de \\ Stefan Napel \\ Dept. of Economics, University of Bayreuth, 95440 Bayreuth, Germany \\ email: stefan.napel@uni-bayreuth.de
}

October 31,2019

\begin{abstract}
Many binary collective choice situations can be described as weighted simple voting games. We introduce weighted committee games to model decisions on an arbitrary number of alternatives in analogous fashion. We compare the effect of different voting weights (shareholdings, party seats, etc.) under plurality, Borda, Copeland, and antiplurality rule. The number and geometry of weight equivalence classes differ widely across the rules. Decisions can be much more sensitive to weights in Borda committees than (anti-)plurality or Copeland ones.
\end{abstract}

Keywords: group decisions and negotiations $\cdot$ weighted voting $\cdot$ simple games $\cdot$ scoring rules $\cdot$ majority rule

We are grateful to two anonymous referees for their constructive comments. This paper has also benefited from suggestions made by several colleagues and conference audiences, especially H. Nurmi, N. Maaser, J. Sobel, and W. Zwicker. 


\section{Introduction}

Consider a corporation of three stockholders owning 6, 5, and 2 million shares. As collective decisions between CEO candidates, new business proposals, etc. are taken, votes are weighted by the respective shareholdings. One may wonder: do the resulting choices differ, ceteris paribus, from those if the three had equal votes? Or, say, from outcomes for a $(48 \%, 24 \%, 28 \%)$ distribution? The answer affects incentives to participate in a capital increase or to invest in the first place. Similar questions arise in international institutions that weight votes by financial contributions or population sizes (IMF, World Bank, EU Council) or when parties cast bloc votes in parliament.

For binary 'yes'-or-'no' decisions and a simple majority requirement, the above distributions of weight are equivalent: any two shareholders jointly meet the majority threshold of 50\%; the different weights induce the same winning coalitions, hence the same binary voting game. This extends to all distributions such that each of three players wields positive but less than half of total weight. A large literature on simple voting games has formalized related results.

However, things differ and much less is known if players choose from three or more options. For instance, the above shareholders may use plurality rule to decide between three CEO candidates. Then investor 1 is decisive whenever 2 and 3 fail to agree: his or her favorite candidate wins with a tally of $6: 5: 2,11: 2$, or $8: 5$ million. Identical plurality winners would result for $(48 \%, 24 \%, 28 \%)$, but ties can arise and yield different decisions for equal votes. The former weights define the same committee game, characterized by $n$ players, $m$ alternatives, and a mapping from $n$-tuples of strict preferences to a winning alternative; equal weights create a different game.

This paper extends several of the - unfortunately, already difficult - questions that the literature has addressed for binary simple games to committee games. Numerical tractability falls sharply in $m$ and $n$ but first answers are feasible. Our main interest lies in equivalence classes of weight distributions for a given group decision rule. One such class, for instance, comprises all weight vectors that induce the same plurality winners as $(6,5,2)$ for decisions on three alternatives. We investigate three scoring rules (plurality, Borda, antiplurality) and one Condorcet rule (Copeland). The respective weight classes can be described by linear inequalities. Monotonicity properties (Felsenthal and Nurmi 2017), degrees of manipulability (Aleskerov and 
Kurbanov 1999), or strategic voting equilibria (Myerson and Weber 1993; Bouton 2013) for one class member directly apply to all.

Inspired by the ongoing quest of characterizing and counting all simple voting games that admit a weighted representation, 11 we try to determine the number of distinct weighted committee games involving either plurality, Borda, antiplurality, or Copeland rule for $m \geq 3$ alternatives. We propose a decisive test, based on integer linear programming, for whether a given choice rule is representable as a weighted instance of a given scoring rule. The superexponential number of $m^{\left(m !^{n}\right)}$ distinct mappings from $n$-tuples of preferences to $m$ potential winners makes the analysis computationally difficult. Still, minimal representations are provided for all 51 Borda committee games with $n=m=3$, all plurality and antiplurality committees with $n \leq 4$, and all Copeland committees with $n \leq 6$ players in the appendix; others are available upon request. Complete enumeration and lists of games can be useful, e.g., to solve the 'inverse problem' of finding a voting game that best achieves a given goal (Kurz 2012b) or to obtain sharp bounds on numbers of players and alternatives that permit a certain monotonicity violation, voting paradox, etc.

The extent to which different voting weights make a real or only a superficial difference has practical relevance. For example, financial drawing rights and thereby voting weights among the 24 Directors of the International Monetary Fund's Executive Board were reformed in 2016. Has the vote change been purely cosmetic or is it possible that the agreed weight increases for emerging market economies affect future decisions, such as the choice of the next IMF Managing Director? The selection process for the latter has been reformed too and involves a shortlist of three candidates that is compiled "taking into account the Fund's weighted voting system" 2 The winner is chosen by consensus or else determined "by a majority of the votes cast" according to the new voting powers. The IMF's weighted voting system would be compatible with pairwise majority comparisons of candidates (Copeland rule) as well as plurality votes in the shortlisting and runoff stages; in the former, use of a scoring method such as Borda rule is conceivable too. Whether different procedural choices at either stage might have an effect and whether the 2016 vote changes could matter for outcomes both boil down to equivalence or not of weighted committee

\footnotetext{
${ }^{1}$ See the monograph by Taylor and Zwicker (1999) and, e.g., Kurz (2012a), Houy and Zwicker (2014). or Freixas et al. (2017). The number of weighted voting games is still unknown for $n>9$.

${ }^{2}$ Cf. www.imf.org/en/About/Factsheets/Managing-Director-Selection-Process/qandas (last accessed: August 12, 2019).
} 
games.

We depict the geometry of weighted committees for $n=3$. The illustrations convey a sense of the (non-)robustness of collective decisions with respect to variations in voting weights that reflect, e.g., changes in corporate voting rights or party switching in parliament. Borda rule can be seen to be highly sensitive to weight variations while collective choice by (anti-)plurality or Copeland rule generates only muted incentives for increasing votes to gain influence on decisions.

\section{Notation and definitions}

\subsection{Preliminaries}

We consider a finite set $N$ of $n \geq 1$ players such that each $i \in N$ has a strict preference relation $P_{i}$ over a set $A=\left\{a_{1}, \ldots, a_{m}\right\}$ of $m \geq 2$ alternatives. $\mathcal{P}(A)$ denotes the set of all $m$ ! strict preference orderings on $A$. A collective choice rule $\rho: \mathcal{P}(A)^{n} \rightarrow A$ maps each profile $\mathbf{P}=\left(P_{1}, \ldots, P_{n}\right)$ to a winning alternative $a^{*}=\rho(\mathbf{P})$. Though $\rho$ is defined on complete preference profiles $\mathbf{P}$, it might draw on partial preference information only and require players, e.g., to submit just the top elements of $P_{1}, \ldots, P_{n}$ in a plurality vote. Note also that $\rho$ does not specify information on how non-winning alternatives $a \neq a^{*}$ are ranked relative to each other: we investigate resolute (singleton-valued) choice rules rather than Arrovian social welfare functions.

Rules $\rho$ that treat all voters $i \in N$ symmetrically play a special role in our analysis: suppose profile $\mathbf{P}^{\prime}=\left(P_{\pi(1)}, \ldots, P_{\pi(n)}\right)$ results from applying a permutation $\pi: N \rightarrow N$ to $\mathbf{P}$. Then $\rho$ is anonymous if $\rho(\mathbf{P})=\rho\left(\mathbf{P}^{\prime}\right)$ for all such $\mathbf{P}, \mathbf{P}^{\prime}$. We write $r$ instead of $\rho$ if we want to highlight that a considered rule is anonymous.

We focus on four standard voting rules. Their definitions are summarized in Table 1 where $b_{i}(a, \mathbf{P}):=\left|\left\{a^{\prime} \in A \mid a P_{i} a^{\prime}\right\}\right|$. Antiplurality rule $r^{A}$, Borda rule $r^{B}$, and plurality rule $r^{P}$ are scoring rules: winners can be characterized as maximizers of scores derived from alternatives' positions in $\mathbf{P}$ and a fixed scoring vector $\mathbf{s} \in \mathbb{Z}^{m}$ with $s_{1} \geq s_{2} \geq \ldots \geq s_{m}$. Namely, let the fact that alternative $a$ is ranked at the $j$-th highest position in ordering $P_{i}$ contribute $s_{j}$ points for $a$, and refer to the sum of all points received as $a^{\prime}$ s score. Then score maximization for $\mathbf{s}^{B}=(m-1, m-2, \ldots, 1,0)$ yields the Borda winner, $\mathbf{s}^{P}=(1,0, \ldots, 0,0)$ the plurality winner, and $\mathbf{s}^{A}=(0,0, \ldots, 0,-1)$ or $(1,1, \ldots, 1,0)$ the antiplurality winner. By contrast, Copeland rule $r^{C}$ considers the 


\begin{tabular}{|l|l|}
\hline Rule & Winning alternative at preference profile $\mathbf{P}$ \\
\hline Antiplurality & $r^{A}(\mathbf{P}) \in \arg \min _{a \in A}\left|\left\{i \in N \mid \forall a^{\prime} \neq a \in A: a^{\prime} P_{i} a\right\}\right|$ \\
Borda & $r^{B}(\mathbf{P}) \in \arg \max _{a \in A} \sum_{i \in N} b_{i}(a, \mathbf{P})$ \\
Copeland & $r^{C}(\mathbf{P}) \in \arg \max _{a \in A}\left|\left\{a^{\prime} \in A \mid a>_{M}^{\mathbf{P}} a^{\prime}\right\}\right|$ \\
Plurality & $r^{P}(\mathbf{P}) \in \arg \max _{a \in A}\left|\left\{i \in N \mid \forall a^{\prime} \neq a \in A: a P_{i} a^{\prime}\right\}\right|$ \\
\hline
\end{tabular}

Table 1: Investigated voting rules

pairwise majority relation $a>_{M}^{\mathbf{P}} a^{\prime}: \Leftrightarrow\left|\left\{i \in N \mid a P_{i} a^{\prime}\right\}\right|>\left|\left\{i \in N \mid a^{\prime} P_{i} a\right\}\right|$ and selects the alternative that beats the most others according to $>_{M}^{\mathbf{P}}$. Clearly, if $>_{M}^{\mathbf{P}}$ has a unique top - the Condorcet winner - then it will be chosen: that is, $r^{C}$ is a Condorcet method.

We impose lexicographic tie breaking. This has computational advantages over working with set-valued choices and entails no loss provided we consider all $\mathbf{P} \in$ $\mathcal{P}(A)^{n}$ : the set of alternatives tied at $\mathbf{P}$ is fully determined by $a^{*}=r(\mathbf{P})$ and the respective winners $a^{* *}, a^{* * *}, \ldots$ at profiles $\mathbf{P}^{\prime}, \mathbf{P}^{\prime \prime}, \ldots$ that swap $a^{*}$ with alternatives $a^{\prime}, a^{\prime \prime}, \ldots$ that might be tied with $a^{*}$ at $\mathbf{P} .^{3}$ Our resolute rules $r^{A}, r^{B}, r^{C}, r^{P}$ and their set-valued versions are hence in one-to-one correspondence and exhibit identical structural equivalences. The same applies to uniform random tie breaking.

We call the combination $(N, A, \rho)$ of a set of voters, a set of alternatives and a collective choice rule a committee game or just a committee. Several special cases have previously received attention in the literature.

Most prominently, committees $(N, A, \rho)$ with binary $A=\{0,1\}$ and surjective, monotonic $\rho$ are known as simple voting games. Following von Neumann and Morgenstern (1953, Ch. 10), these games are usually described as a pair $(N, v)$ with $v: 2^{N} \rightarrow\{0,1\}$ and $v(S)=1$ when $1 P_{i} 0$ for all $i \in S$ implies $\rho(\mathbf{P})=1$. Sets $S \subseteq N$ with $v(S)=1$ are known as winning coalitions. $(N, v)$ is weighted and called weighted voting game if there exists a non-negative vector $\mathbf{w}=\left(w_{1}, \ldots, w_{n}\right)$ of weights and a positive quota $q$ such that $v(S)=1$ if and only if $\sum_{i \in S} w_{i} \geq q !^{4}$ Pair $(q ; \mathbf{w})$ is a (weighted) representation of $(N, v)$ and one writes $(N, v)=[q ; \mathbf{w}]$. It is no restriction to focus on integers: given $q \in \mathbb{R}_{++}, \mathbf{w} \in \mathbb{R}_{+}^{n}$ there always exist $q^{\prime} \in \mathbb{N}, \mathbf{w}^{\prime} \in \mathbb{N}_{0}^{n}$ such that $[q ; \mathbf{w}]=\left[q^{\prime} ; \mathbf{w}^{\prime}\right]$.

${ }^{3}$ Given $r(\mathbf{P})=b$, for example, a tie with $a$ can directly be ruled out; one sees if $b$ was tied with $c$ by checking whether $r\left(\mathbf{P}^{\prime}\right)=c$ or $b$ where $\mathbf{P}^{\prime}$ only swaps $b^{\prime}$ s and $c^{\prime}$ s position in every player's ranking $P_{i}$.

${ }^{4}$ Existence is guaranteed only for $n \leq 3$. Games that are not weighted arise, e.g., in the Legislative Council of Hong Kong (Cheung and Ng 2014) and the EU Council (Kurz and Napel 2016). 
Other special cases include voting with multiple levels of approval, such as ternary voting games (Felsenthal and Machover 1997), quaternary voting games (Laruelle and Valenciano 2012), and $(j, k)$-games (Hsiao and Raghavan 1993; Freixas and Zwicker 2003). Plurality committees (defined below) have featured in the multicandidate voting frameworks of Bolger (1983), Amer et al. (1998) and Monroy and Fernández (2009. 2011) as simple plurality games and relative majority r-games 5

\subsection{Weighted committee games}

Many committee games that model real collective decision making involve a nonanonymous rule $\rho$. For instance, an anonymous decision rule $r$ may apply at the level of shareholdings, IMF drawing rights, etc. rather than that of individual voters; or players $i \in N$ are well-disciplined parties with different numbers of seats. ${ }^{6}$ The corresponding rule $\rho$ can be viewed as the combination of an anonymous collective choice rule $r$ with integer voting weights $w_{1}, \ldots, w_{n}$ attached to the players.

With $r$ denoting the entire family of mappings from $n$-tuples of linear orders over $A$ to winners $a^{*} \in A$ under the considered rule, we define $r \mid \mathbf{w}: \mathcal{P}(A)^{w_{\Sigma}} \rightarrow A$ by

$$
r \mid \mathbf{w}(\mathbf{P}):=r(\underbrace{P_{1}, \ldots, P_{1}}_{w_{1} \text { times }}, \underbrace{P_{2}, \ldots, P_{2}}_{w_{2} \text { times }}, \ldots, \underbrace{P_{n}, \ldots, P_{n}}_{w_{n} \text { times }})
$$

for a given anonymous rule $r$ and a non-negative, non-degenerate weight vector $\mathbf{w}=\left(w_{1}, \ldots, w_{n}\right) \in \mathbb{N}_{0}^{n}$ with $w_{\Sigma}:=\sum_{i=1}^{n} w_{i}>0$. In the degenerate case $\mathbf{w}=(0, \ldots, 0)$, let $r \mid \mathbf{0}(\mathbf{P}) \equiv a_{1}$.

We say a committee game $(N, A, \rho)$ is $r$-weighted for a given rule $r$ if there exists a weight vector $\mathbf{w}=\left(w_{1}, \ldots, w_{n}\right) \in \mathbb{N}_{0}^{n}$ such that $(N, A, \rho)=(N, A, r \mid \mathbf{w})$, i.e.,

$$
\rho(\mathbf{P})=r \mid \mathbf{w}(\mathbf{P}) \text { for all } \mathbf{P}=\left(P_{1}, \ldots, P_{n}\right) \in \mathcal{P}(A)^{n} .
$$

$(N, A, r, \mathbf{w})$ is a (weighted) representation of $(N, A, \rho)$ and we also denote this game by $[N, A, r, \mathbf{w}] .\left[N, A, r^{A}, \mathbf{w}\right],\left[N, A, r^{B}, \mathbf{w}\right],\left[N, A, r^{C}, \mathbf{w}\right]$ and $\left[N, A, r^{P}, \mathbf{w}\right]$ are referred to as

\footnotetext{
${ }^{5}$ Their analysis focused on power indices. We here show that there are only 36 distinct simple plurality games with four players and so at most 36 different distributions of power can arise.

${ }^{6}$ If designated members enjoy procedural privileges, this may - but need not - be equivalent to asymmetric weights. Veto power of permanent members in the UN Security Council, e.g., translates into $[39 ; 7,7,7,7,7,1, \ldots, 1]$ for $m=2$.
} 


\begin{tabular}{|c|c|c|c|c|c|c|}
\hline$P_{1}$ & $P_{2}$ & $P_{3}$ & $P_{4}$ & \multirow{5}{*}{$\Rightarrow$} & & \\
\hline$d$ & $b$ & $c$ & $c$ & & $r^{A} \mid \mathbf{w}(\mathbf{P})=a$ & ( $a$ has min. negative votes 0 ) \\
\hline$e$ & $c$ & $e$ & $b$ & & $r^{B} \mid \mathbf{w}(\mathbf{P})=b$ & ( $b$ has max. Borda score 28 ) \\
\hline$b$ & $e$ & $a$ & $a$ & & ${ }^{C} \mid \mathbf{w}(\mathbf{P})=c$ & ( $c$ has max. pairwise wins 3 ) \\
\hline$a$ & $a$ & $d$ & $d$ & & $r^{P} \mid \mathbf{w}(\mathbf{P})=d$ & ( $d$ has max. plurality tally 5 ) \\
\hline$c$ & $d$ & $b$ & $e$ & & & \\
\hline
\end{tabular}

Table 2: Choices for preference profile $\mathbf{P}$ when $\mathbf{w}=(5,3,2,2)$

antiplurality, Borda, Copeland and plurality committees respectively. Such committees typically differ for $m>2$, as illustrated in Table 2 the winner from $A=\{a, b, c, d, e\}$ at $\mathbf{P}$ all depends on the voting rule in use. Neither this observation nor below structural findings depend on whether $\mathbf{P}$ reflects sincere or strategic preference statements.

By definition (1) uniform weights $\mathbf{w}=(1, \ldots, 1)$ reduce any collective choice rule $r \mid \mathbf{w}$ to $r$. It follows that monotonicity, consistency, and other properties that are satisfied (violated) by a given rule $r$ are also satisfied (violated) by $r \mid \mathbf{w}$. For instance, the axiomatic characterizations by Young (1975) of set-valued versions of general scoring rules, Henriet (1985) of a Copeland variation, or Kurihara (2018) of antiplurality continue to apply. Non-uniform weights $\mathbf{w}$ essentially impose the domain restriction that $w_{1}, w_{2}, \ldots$ individuals have identical preferences. At a given profile $\mathbf{P}$, a group $i$ of voters can be worse off under $r \mid \mathbf{w}^{\prime}$ than $r \mid \mathbf{w}$ for $w_{i}^{\prime}>w_{i}$ and $w_{j}^{\prime}=w_{j}, j \neq i$, if and only if $r$ suffers from the so-called no show paradox. Condorcet methods like Copeland rule are known to do so (see Moulin 1988), while scoring rules do not. Our question here is related but a broader one: for given $r$, which weight variations $\mathbf{w}^{\prime} \neq \mathbf{w}$ can or cannot induce choice changes compared to $r \mid \mathbf{w}$ ?

\section{Equivalence classes of weighted committee games}

\subsection{Equivalence of committee games}

Two $r$-weighted committee games $(N, A, r \mid \mathbf{w})$ and $\left(N^{\prime}, A^{\prime}, r \mid \mathbf{w}^{\prime}\right)$ are structurally equivalent or equivalent up to isomorphism if

$$
\left\{a_{j} P_{i} a_{k} \Leftrightarrow \tilde{\pi}\left(a_{j}\right) P_{\pi(i)}^{\prime} \tilde{\pi}\left(a_{k}\right)\right\} \Rightarrow \tilde{\pi}(r \mid \mathbf{w}(\mathbf{P}))=r \mid \mathbf{w}^{\prime}\left(\mathbf{P}^{\prime}\right)
$$


for bijections $\pi: N \rightarrow N^{\prime}$ and $\tilde{\pi}: A \rightarrow A^{\prime}$ that map every profile $\mathbf{P}$ of preferences $P_{i}$ over $A$ to a relabeled profile $\mathbf{P}^{\prime}$ of preferences $P_{\pi(i)}^{\prime}$ over $A^{\prime}, 7$ For instance, Copeland committees with $N=N^{\prime}=\{1,2,3\}, A=A^{\prime}, \mathbf{w}=(3,1,1)$ and $\mathbf{w}^{\prime}=(1,3,1)$ have quite different attractiveness to player 1 but the decision environment is structurally the same: there is a dictator player whose most-preferred alternative wins and two null players whose preferences do not affect the outcome.

A given distribution $\mathbf{w} \in \mathbb{N}_{0}^{n}$ fixes $n$ and we can write $(r, \mathbf{w}) \sim_{m}\left(r, \mathbf{w}^{\prime}\right)$ if $r$ committee games with $m$ alternatives are structurally equivalent for weights $\mathbf{w}$ and $\mathbf{w}^{\prime}$. Relation $\sim_{m}$ and some $\overline{\mathbf{w}} \in \mathbb{N}_{0}^{n}$ with $\bar{w}_{1} \geq \bar{w}_{2} \geq \ldots \geq \bar{w}_{n}$ define the equivalence class

$$
\mathcal{E}_{\overline{\mathbf{w}}, m}^{r}:=\left\{\mathbf{w} \in \mathbb{N}_{0}^{n} \mid(r, \mathbf{w}) \sim_{m}(r, \overline{\mathbf{w}})\right\} .
$$

If rule $r$ is in use for deciding between $m$ alternatives, then all weight distributions $\mathbf{w}, \mathbf{w}^{\prime} \in \mathcal{E}_{\overline{\mathbf{w}}, m}^{r}$ come with identical monotonicity properties, voting paradoxes, manipulation incentives, implementation possibilities, strategic equilibria, etc.

\subsection{Illustration}

As an example, consider Borda rule for $m=3$ and reference weights $\overline{\mathbf{w}}=(5,2,1)$ that reflect a given seat distribution in a council, voting stocks held by shareholders, etc. We focus on the subset $\mathcal{E}_{(5,2,1), 3}^{B} \subset \mathcal{E}_{(5,2,1), 3}^{r^{B}}$ of alternative distributions w with $w_{1} \geq w_{2} \geq w_{3}$. Two linear inequalities are implied by $r^{B}\left|(5,2,1)=r^{B}\right| \mathbf{w}$ for each profile $\mathbf{P} \in \mathcal{P}(A)^{3}$. For instance, writing $a b c$ in abbreviation of $a P_{i} b P_{i} c$, profile $\mathbf{P}=(c a b, b a c, a b c)$ gives rise to a total Borda score $\bar{w}_{1} \cdot 1+\bar{w}_{2} \cdot 1+\bar{w}_{3} \cdot 2=9$ for alternative $a$ : it is ranked top by player 3 , middle by players 1 and 2 . The corresponding scores for $b$ and $c$ are $2 \bar{w}_{2}+\bar{w}_{3}=5$ and $2 \bar{w}_{1}=10$. Hence $r^{B} \mid \overline{\mathbf{w}}(\mathbf{P})=c$ and any allocation $\mathbf{w}$ of seats, shares, etc. that is equivalent to $\overline{\mathbf{w}}$ must ensure that the respective Borda score $2 w_{1}$ of (lexicograpically maximal) $c$ strictly exceeds $a^{\prime}$ s and $b^{\prime}$ s scores:

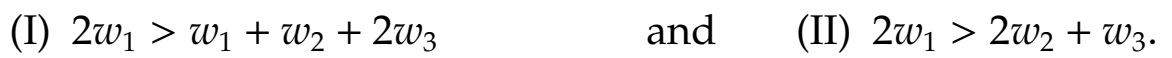

\footnotetext{
${ }^{7}$ Analogous equivalences apply to $(N, A, r \mid \mathbf{w})$ and $\left(N^{\prime}, A^{\prime}, r^{\prime} \mid \mathbf{w}^{\prime}\right)$ when $r \neq r^{\prime}$ or general committees $(N, A, \rho)$ and $\left(N^{\prime}, A^{\prime}, \rho^{\prime}\right)$. The respective considerations could, in principle, also be extended to Arrovian social welfare functions, which map each profile of individual preferences to a collective preference $P^{*} \in \mathcal{P}(A)$ rather than a winning alternative $a^{*} \in A$. We leave detailed explorations to future research.
} 
$\mathbf{P}^{\prime}=(c a b, a b c, b a c)$ makes $a$ the winner. Its score must not be smaller than $b^{\prime}$ s and $c^{\prime} \mathbf{s}$ :

$$
\text { (III) } w_{1}+2 w_{2}+w_{3} \geq w_{2}+2 w_{3} \quad \text { and } \quad \text { (IV) } w_{1}+2 w_{2}+w_{3} \geq 2 w_{1} \text {. }
$$

Wins by $a$ and $b$ for $\mathbf{P}^{\prime \prime}=(a b c, b c a, b a c)$ and $\mathbf{P}^{\prime \prime \prime}=(a b c, b c a, b c a)$ similarly imply:

$$
\begin{array}{lll}
\text { (V) } 2 w_{1}+w_{3} \geq w_{1}+2 w_{2}+2 w_{3} & \text { and } & \text { (VI) } 2 w_{1}+w_{3} \geq w_{2} \\
\text { (VII) } w_{1}+2 w_{2}+2 w_{3}>2 w_{1} & \text { and } & \text { (VIII) } w_{1}+2 w_{2}+2 w_{3} \geq w_{2}+w_{3} .
\end{array}
$$

Condition (VIII) is trivially satisfied. (IV) and (V) imply $w_{1}=2 w_{2}+w_{3}$. This makes (I) equivalent to $w_{2}>w_{3}$ and (VII) to $w_{3}>0$. Combining $w_{1}=2 w_{2}+w_{3}$ and $w_{2}>w_{3}>0$ also verifies (II), (III) and (VI). The 212 remaining profiles $\mathbf{P} \in \mathcal{P}(A)^{3}$ turn out not to impose additional constraints. Hence

$$
\mathbf{w} \in \mathcal{E}_{(5,2,1), 3}^{B}=\left\{\left(2 w_{2}+w_{3}, w_{2}, w_{3}\right) \in \mathbb{N}_{0}^{3}: w_{2}>w_{3}>0\right\}
$$

contains all weight distributions $w_{1} \geq w_{2} \geq w_{3}$ that imply Borda choices identical to $\overline{\mathbf{w}}=(5,2,1)$ for all preference profiles over three options. The full class $\mathcal{E}_{(5,2,1), 3}^{r^{B}}$ follows by permuting the distributions in $\mathcal{E}_{(5,2,1), 3}^{B}$. Other classes, such as $\mathcal{E}_{(1,1,1), 3^{\prime}}^{r^{B}} \mathcal{E}_{(2,1,1), 3^{\prime}}^{r^{3}}$ etc., are characterized by analogous inequalities.

\subsection{Relation between equivalence classes}

The number of distinct mappings from preference profiles to outcomes is large but finite for given $n$ and $m$. $\mathbb{N}_{0}^{n}$ hence is partitioned into a finite collection $\left\{\mathcal{E}_{\overline{\mathbf{w}}_{1}, m}^{r}, \mathcal{E}_{\overline{\mathbf{w}}_{2}, m^{\prime}}^{r}\right.$ $\left.\ldots, \mathcal{E}_{\overline{\mathrm{w}}_{\xi}, m}^{r}\right\}$ for any given rule $r$. We will investigate numerically how the number $\xi$ of elements varies across rules but let us first state some analytical observations. The first two are obvious:

Proposition 1. The partitions $\left\{\mathcal{E}_{\overline{\mathrm{w}}_{1}, 2}^{r}, \ldots, \mathcal{E}_{\overline{\mathrm{w}}_{\xi}, 2}^{r}\right\}$ of $\mathbb{N}_{0}^{n}$ coincide for $r \in\left\{r^{A}, r^{B}, r^{C}, r^{P}\right\}$.

Proposition 2. Let $A=\left\{a_{1}, a_{2}\right\}$ and $r \in\left\{r^{A}, r^{B}, r^{C}, r^{P}\right\}$. Then $r \mid \mathbf{w}(\mathbf{P})=a_{1} \Leftrightarrow v(S)=1$ where $(N, v)=[q ; \mathbf{w}]$ with $q=\frac{1}{2} \sum_{i \in N} w_{i}$ and $S=\left\{i \in N \mid a_{1} P_{i} a_{2}\right\}$.

It follows that the respective partitions $\left\{\mathcal{E}_{\overline{\mathbf{w}}_{1}, 2}^{r}, \ldots, \mathcal{E}_{\overline{\mathbf{w}}_{\xi, 2}}^{r}\right\}$ of $\mathbb{N}_{0}^{n}$ coincide with those for weighted voting games with a simple majority quota. Their study and enumeration for $n \leq 5$ dates back to von Neumann and Morgenstern (1953, Ch. 10). 
The next observations vary $m$ for fixed $r$; proofs are given in Appendix A:

Proposition 3. The antiplurality partitions $\left\{\mathcal{E}_{\overline{\mathbf{w}}_{1}, m}^{r^{A}}, \mathcal{E}_{\overline{\mathbf{w}}_{2}, m^{\prime}}^{r^{A}}, \ldots, \mathcal{E}_{\overline{\mathbf{w}}_{\xi}, m}^{r^{A}}\right\}$ of $\mathbb{N}_{0}^{n} \backslash\{\mathbf{0}\}$ consist of $\xi=n$ equivalence classes identified by weight vectors $\overline{\mathbf{w}}_{\mathbf{1}}=(1,0, \ldots, 0), \overline{\mathbf{w}}_{\mathbf{2}}=$ $(1,1, \ldots, 0), \ldots, \overline{\mathbf{w}}_{\mathbf{n}}=(1,1, \ldots, 1)$ for all $m \geq n+1$.

Proposition 4. For Borda rule $r^{B}$ and $m \geq 3$, every weight vector $\tilde{\mathbf{w}}_{\mathbf{j}}=(j, 1,0, \ldots, 0)$ with $j \in\{1, \ldots, m-1\}$ identifies a different class $\mathcal{E}_{\tilde{\mathrm{w}}_{\mathrm{j}}, m}^{r^{B}}$.

This implies that - differently from antiplurality, Copeland, and plurality - the number $\xi$ of structurally distinct Borda committee games for given $n \geq 3$ grows in $m$ without bound.

Proposition 5. The Copeland partitions $\left\{\mathcal{E}_{\overline{\mathbf{w}}_{1}, m}^{r^{C}}, \ldots, \mathcal{E}_{\overline{\mathbf{w}}_{\xi}, m}^{r^{c}}\right\}$ of $\mathbb{N}_{0}^{n}$ coincide for all $m \geq 2$.

So $r^{C}$ extends the known equivalences for binary simple voting games to arbitrarily many options. This might feel unsurprising because winners in Copeland committees are selected by binary comparisons.

However, the conjecture that Prop. 5 applies to just any Condorcet method is wrong. Copeland rule is special. For instance, Black rule selects the Condorcet winner if one exists and otherwise breaks cyclical majorities by Borda scores. Weight distributions of $(6,4,3)$ and $(4,4,2)$ are equivalent for $m=2$ and give rise to a cycle $a>_{M}^{\mathbf{P}} b>_{M}^{\mathbf{P}} c>_{M}^{\mathbf{P}} a$ for $\mathbf{P}=(c a b, a b c, b c a)$. The Black winner then is $c$ for the former but $a$ for the latter weights; so they are non-equivalent for $m=3$. The same applies to Kemeny-Young or maximum likelihood rule, which picks the top element of the collective preference ranking $P^{*}$ that minimizes total pairwise disagreements with all individual rankings in $\mathbf{P}$; or maximin rule, where the winner maximizes the minimum support across all pairwise comparisons.

Proposition 6. The plurality partitions $\left\{\mathcal{E}_{\overline{\mathrm{w}}_{1}, m^{p}}^{r^{p}}, \ldots, \mathcal{E}_{\overline{\mathrm{w}}_{\xi}, m}^{r^{p}}\right\}$ of $\mathbb{N}_{0}^{n}$ coincide for all $m \geq n$.

\section{Identification of weighted committees}

\subsection{Minimal representations and test for weightedness}

Given $(N, A, \rho)=(N, A, r \mid \mathbf{w})$, we say that $(N, A, r, \mathbf{w})$ has minimum integer sum or is a minimal representation of $(N, A, \rho)$ if $\sum_{i \in N} w_{i}^{\prime} \geq \sum_{i \in N} w_{i}$ for all representations 
$\left(N, A, r, \mathbf{w}^{\prime}\right)$ of $(N, A, \rho)$. Games in a given equivalence class $\mathcal{E}_{\overline{\mathbf{w}}, m}^{r}$ usually have a unique minimal representation $\sqrt{8}$ corresponding weights are a focal choice for $\overline{\mathbf{w}}$. Minimal representations can be more informative or convenient to work with than given weights in applications (cf. Freixas and Kaniovski 2014, Kurz and Napel 2016).

Finding minimal representations of all Copeland committees simplifies to finding weighted representations of simple voting games (Prop. 2 and 5). Linear programming techniques have proven useful there and can be adapted to scoring rules $r^{A}$, $r^{B}$, or $r^{P}$. Namely, consider a scoring rule $r$ based on an arbitrary but fixed scoring vector s. Write $S_{k}\left(P_{i}\right) \in \mathbb{Z}$ for the unweighted $\left(s_{1}, s_{2}, \ldots, s_{m}\right)$-score of alternative $a_{k}$ derived from its position in ordering $P_{i}$; for instance, for $m=3$ and $a_{3}=c$, we have $S_{3}\left(P_{i}\right)=s_{2}$ if either $a P_{i} c P_{i} b$ or $b P_{i} c P_{i} a$. Now suppose that $r$ can be combined with integer voting weights so as to induce choice rule $\rho$. Then - denoting the index of the winning alternative at profile $\mathbf{P}$ by $\omega_{\rho}(\mathbf{P}) \in\{1, \ldots, m\}$, i.e., $\rho(\mathbf{P})=a_{\omega_{\rho}(\mathbf{P})} \in A-$ any solution to the following integer linear program yields a minimal representation $(N, A, r, \mathbf{w})$ of $(N, A, \rho)$ :

$$
\begin{array}{ll} 
& \min _{\mathbf{w} \in \mathbb{N}_{0}^{n}} \sum_{i=1}^{n} w_{i} \\
\text { s.t. } & \sum_{i=1}^{n} S_{k}\left(P_{i}\right) \cdot w_{i} \leq \sum_{i=1}^{n} S_{\omega_{\rho}(\mathbf{P})}\left(P_{i}\right) \cdot w_{i}-1 \quad \forall \mathbf{P} \in \mathcal{P}(A)^{n} \forall 1 \leq k \leq \omega_{\rho}(\mathbf{P})-1, \\
& \sum_{i=1}^{n} S_{k}\left(P_{i}\right) \cdot w_{i} \leq \sum_{i=1}^{n} S_{\omega_{\rho}(\mathbf{P})}\left(P_{i}\right) \cdot w_{i} \quad \forall \mathbf{P} \in \mathcal{P}(A)^{n} \forall \omega_{\rho}(\mathbf{P})+1 \leq k \leq m .
\end{array}
$$

The case distinction between alternatives $a_{k}$ with index $k<\omega_{\rho}(\mathbf{P})$ vs. $k>\omega_{\rho}(\mathbf{P})$ reflects the tie breaking assumption. If some (non-minimal) representation $\left(N, A, r, \mathbf{w}^{\prime}\right)$ of $(N, A, \rho)$ is known and $w_{1}^{\prime} \geq w_{2}^{\prime} \geq \ldots \geq w_{n}^{\prime}$ then adding constraints $w_{i} \geq w_{i+1}, \forall 1 \leq i \leq$ $n-1$, to (ILP) accelerates computations.

If it is not known whether $\rho$ is $r$-weighted, (ILP) provides a decisive test for $r$ weightedness for any given scoring rule $r$ (and $r^{C}$ by Prop. 1 and 5): the constraints characterize a non-empty compact set if and only if $\rho$ is $r$-weighted. Checking nonemptiness of (ILP)'s constraint set can be done with software (e.g., Gurobi or CPLEX) that determines a weight sum minimizer at little extra effort.

\footnotetext{
${ }^{8}$ For $m=2$, minimal representations are unique up to $n=7$ players (Kurz 2012a). Multiplicities for games with larger values of $m$ or $n$ arise but are rare.
} 


\section{Branch-and-Cut Algorithm}

Given $n, m$ and $r$, identify every class $\mathcal{E}_{\overline{\mathbf{w}}_{\mathrm{k}}, m}^{r}$ by a minimal representation.

Step 1 Generate all $J:=(m !)^{n}$ profiles $\mathbf{P}^{1}, \ldots, \mathbf{P}^{J} \in \mathcal{P}(A)^{n}$ for $A:=\left\{a_{1}, \ldots, a_{m}\right\}$. Set $\mathcal{F}:=\varnothing$.

Step 2 For every $\mathbf{P}^{j} \in \mathcal{P}(A)^{n}$ and every $a_{i} \in A$, check if there is any weight vector $\mathbf{w} \in \mathbb{N}_{0}^{n}$ s.t. $r \mid \mathbf{w}\left(\mathbf{P}^{j}\right)=a_{i}$ by testing feasibility of the implied constraints (cf. Section 3.2). If yes, then append $(i, j)$ to $\mathcal{F}$.

Step 3 Loop over $j$ from 1 to $J$.

Step 3a If $j=1$, then set $C_{1}:=\{1 \leq i \leq m \mid(i, j) \in \mathcal{F}\}$.

Step $3 \mathbf{b}$ If $j \geq 2$, then set $C_{j}:=\varnothing$ and loop over all $\left(p_{1}, \ldots, p_{j-1}\right) \in C_{j-1}$ and all $p_{j} \in\{1, \ldots, m\}$ with $\left(p_{j}, j\right) \in \mathcal{F}$. If (ILP) has a solution for the restriction to the profiles $\mathbf{P}^{\mathbf{1}}, \ldots, \mathbf{P}^{\mathbf{j}}$ with prescribed winners $\rho\left(\mathbf{P}^{\mathbf{i}}\right)=$ $a_{p_{i}}$ for $1 \leq i \leq j$, then append $\left(p_{1}, \ldots, p_{j}\right)$ to $C_{p}$.

Step 4 Loop over the elements $\left(p_{1}, \ldots, p_{j}, \ldots, p_{J}\right) \in C_{J}$ and output minimal weights $\overline{\mathbf{w}}$ such that $r \mid \overline{\mathbf{w}} \equiv \rho$ with $\rho\left(\mathbf{P}^{\mathbf{j}}\right)=p_{j}$ by solving (ILP).

Table 3: Determining the classes of $r$-weighted committees for given $n$ and $m$

\subsection{Algorithm for identifying all $r$-committees}

In principle, one could find and characterize all distinct $r$-committee games for fixed $n$ and $m$ as follows: loop over all $m^{\left(m !^{n}\right)}$ mappings $\rho: \mathcal{P}(A)^{n} \rightarrow A$; conduct above test; in case of success, determine a representation $(N, A, r, \overline{\mathbf{w}})$ and characterize $\mathcal{E}_{\overline{\mathbf{w}}, m}^{r}$ as in Section 3.2; continue until all choice rules $\rho$ have been covered.

The explosive growth of $m^{\left(m !^{n}\right)}$ prevents a direct implementation of this idea. 9 However, many mappings can be dropped from consideration. If $\rho(\mathbf{P})=a_{1}$ for one of the $(m-1) !^{n}$ profiles $\mathbf{P}$ where $a_{1}$ is unanimously ranked last, for instance, then $\rho$ cannot be $r$-weighted for $r \in\left\{r^{A}, r^{B}, r^{C}, r^{P}\right\}$. This rules out $m^{\left(m !^{n}-1\right)}$ candidate mappings in one go. Similarly, if weights $\mathbf{w}$ such that $r \mid \mathbf{w}(\mathbf{P})=a_{1}$ turn out to be incompatible with $r \mid \mathbf{w}\left(\mathbf{P}^{\prime}\right)=a_{2}$ for two suitable profiles $\mathbf{P}, \mathbf{P}^{\prime}$, then all $m^{\left(m !^{n}-2\right)}$ mappings $\rho$ with $\rho(\mathbf{P})=a_{1}$ and $\rho\left(\mathbf{P}^{\prime}\right)=a_{2}$ can be disregarded at once. The branch-and-cut algorithm described in Table 3 operationalizes these considerations.

The algorithm can still require impractical memory size and running time. The main alternative then is to heuristically loop over different weight distributions and check if they are structurally distinct from those already known. Namely, start with

\footnotetext{
${ }^{9} 3^{\left(3 !^{3}\right)}=3^{216}>10^{103}$ already exceeds the estimated number of atoms in the universe.
} 
$w_{\Sigma}:=0$ and an empty list $\hat{W}$ of weight vectors; increase the sum of weights $w_{\Sigma}$ in

steps of 1 ; generate the set $\mathcal{W}_{w_{\Sigma}}:=\left\{\mathbf{w} \in \mathbb{N}_{0}^{n} \mid w_{1} \geq \cdots \geq w_{n}\right.$ and $\left.w_{1}+\cdots+w_{n}=w_{\Sigma}\right\}$ and loop over all $\mathbf{w} \in \mathcal{W}_{w_{\Sigma}}$. The respective weight vector $\mathbf{w}$ is appended to $\hat{\mathcal{W}}$ if for every $\mathbf{w}^{\prime} \in \hat{\mathcal{W}}$ we have $r|\mathbf{w}(\mathbf{P}) \neq r| \mathbf{w}^{\prime}(\mathbf{P})$ for at least one $\mathbf{P} \in \mathcal{P}(A)^{n}$. The set $\hat{\mathcal{W}}$ then contains a growing list of minimal weight vectors that induce different mappings from preference profiles to winners and hence correspond to structurally distinct committee games $[N, A, r, \mathbf{w}]$. This method has the advantage of not requiring a weightedness test, such as (ILP). However, search needs to be stopped manually and just produces a lower bound on the actual number of classes ${ }^{10}$

\section{Number and geometry of weighted committee games}

\subsection{Number of antiplurality, Borda, Copeland, and plurality games}

A combination of our analytical observations and computational means permits identification of all structurally distinct $r$-weighted committee games with $r \in\left\{r^{A}, r^{B}, r^{C}, r^{P}\right\}$ for small $n$ and $m$. Table 4 summarizes our findings; figures do not include $\mathcal{E}_{0, m} .{ }^{11}$ The branch-and-cut approach required excessive memory for Borda committees when $m>4$ or $n=m \geq 4 .{ }^{12}$ We write " $\geq \ldots$ " if the heuristic appended no new games to set $\hat{W}$ for long enough to support the conjecture that the reported bound equals the exact number of games; we write " $\gg$..." otherwise.

Figures for $m=2$ and $n \leq 6$ have been obtained in the literature before; the others are, to our knowledge, new. When less than 150 equivalence classes of games exist, we report minimal representations in Appendix B. Our list for $m=2$ nests the weighted voting games with 50\%-majority threshold reported by Krohn and Sudhölter (1995) and Brams and Fishburn (1996); plurality committees with $m=3$ nest the subset of tie-free games identified by Chua et al. (2002) for $n=3,4$.

\footnotetext{
${ }^{10}$ Upper bounds exist for weight sums that guarantee coverage of all equivalence classes (cf. Muroga 1971. Thm. 9.3.2.1). The bounds are too large to be practical, however.

${ }^{11} \mathbf{w}^{0}=\mathbf{0}$ always forms its own equivalence class: consider the unanimous profile $\mathbf{P}=(P, \ldots, P) \in$ $\mathcal{P}(A)^{n}$ with $a_{2} P a_{3} P \ldots P a_{m} P a_{1}$. Then $r \mid \mathbf{0}(\mathbf{P})=a_{1}$ but $r \mid \mathbf{w}(\mathbf{P})=a_{2}$ for any $\mathbf{w} \neq \mathbf{0}$.

${ }^{12}$ We used 128 GB RAM and eight $3.0 \mathrm{GHz}$ cores. Several instances ran for more than six months.
} 


\begin{tabular}{|c|c|c|c|c|}
\hline 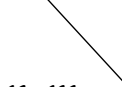 & Antiplurality & Borda & Copeland & Plurality \\
\hline 3,2 & \multicolumn{4}{|c|}{4} \\
\hline 4,2 & \multicolumn{4}{|c|}{9} \\
\hline 5,2 & \multicolumn{4}{|c|}{27} \\
\hline 6,2 & \multicolumn{4}{|c|}{138} \\
\hline 7,2 & \multicolumn{4}{|c|}{1663} \\
\hline 8,2 & \multicolumn{4}{|c|}{63764} \\
\hline 9,2 & \multicolumn{4}{|c|}{9425479} \\
\hline 3,3 & 5 & 51 & 4 & 6 \\
\hline 3,4 & 3 & 505 & 4 & 6 \\
\hline 3,5 & 3 & $\geq 2251$ & 4 & 6 \\
\hline 4,3 & 19 & 5255 & 9 & 34 \\
\hline 4,4 & 7 & $\gg 635622$ & 9 & 36 \\
\hline 4,5 & 4 & $\gg 635622$ & 9 & 36 \\
\hline 5,3 & 263 & $\gg 1153448$ & 27 & 852 \\
\hline 6,3 & $\geq 33583$ & $\gg 1153448$ & 138 & $\gg 147984$ \\
\hline
\end{tabular}

Table 4: Numbers of distinct weighted committee games 
(a)

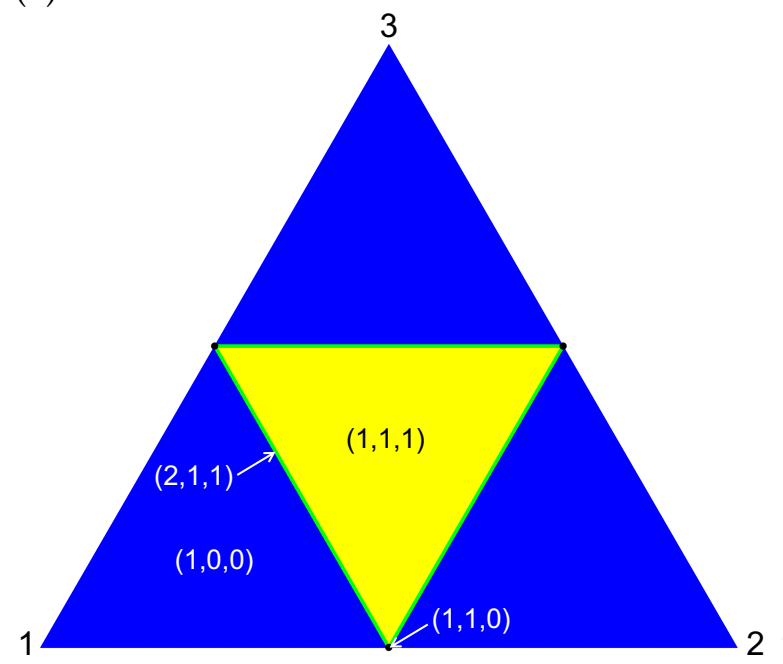

(b)

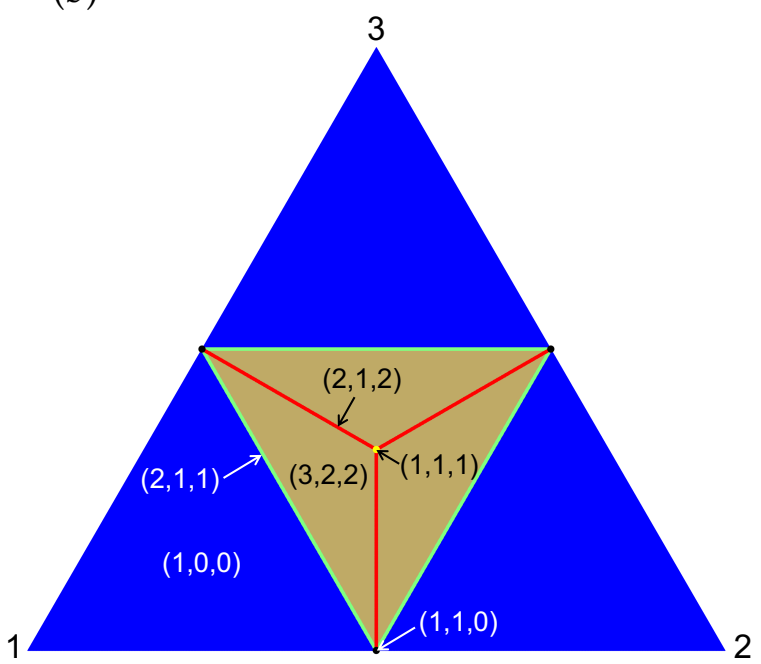

Figure 1: The four Copeland and six plurality equivalence classes

\subsection{Geometry of committee games with $n=3$}

The case of three players allows to convey a geometric idea of how robust a given weighted committee is to small changes in weights. Our illustrations echo those by Saari (1995, 2001): his eponymous triangles concern $m=3$ alternatives and arbitrary numbers $n$ of individual voters. They illuminate how collective rankings vary with the applicable voting procedure for fixed preferences $\mathbf{P}$. We, by contrast, assume $n=3$ voter blocs, evaluate all preferences, and the number $m$ of alternatives may vary. We use the standard projection of the 3-dimensional unit simplex of relative weights to the plane. Points of identical color correspond to structurally equivalent weight distributions, i.e., they induce isomorphic committee games for the investigated voting rule $r$. When equivalence classes are line segments or single points, we have manually enlarged them in Figures 1 and 2 to improve visibility.

Figure 1(a) shows all Copeland committees with three players and their minimal representations. Very dissimilar weight distributions like $(33,33,33)$ and $(49,49,1)$ induce the same Copeland winners. Figure 1(b) illustrates that plurality rule is more sensitive to weight perturbations than Copeland rule, at least for non-dictatorial configurations. This is more pronounced the more voter blocs are involved: there are about four and 32 times more distinct committees with plurality than Copeland rule for $n=4$ and 5 (Table 4); we conjecture the factor exceeds 1000 for $n=6$.

Antiplurality rule (Figure 2), characterized recently with new axioms by Kurihara 
(a) $m=3$

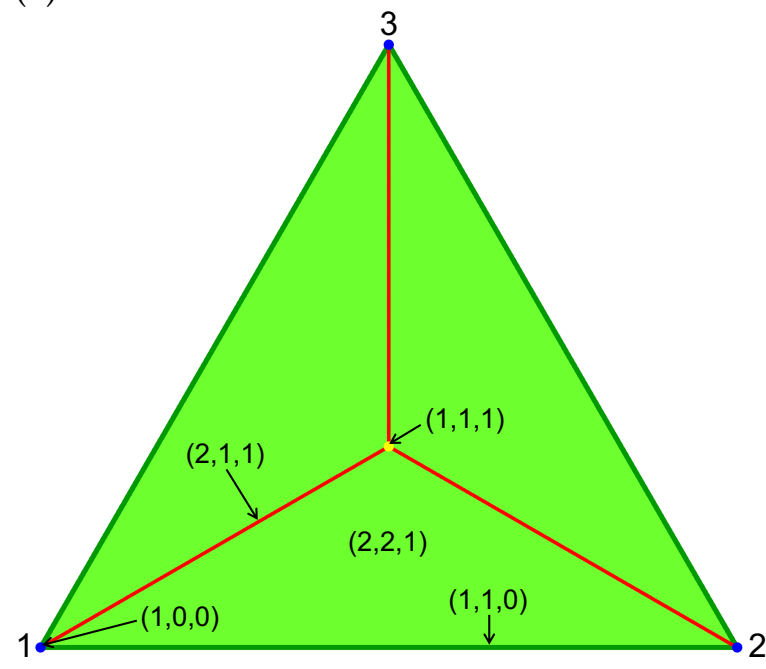

(b) $m \geq 4$

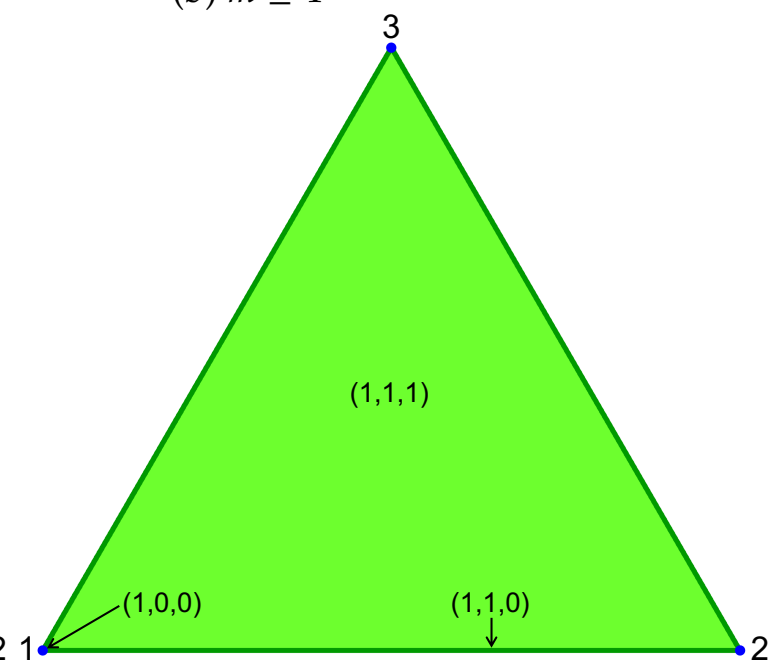

Figure 2: The five or three antiplurality equivalence classes

(2018), holds an intermediate ground in terms of the sensitivity to $\mathbf{w}$. The most scope for changes in the distribution of voting rights to induce different decisions comes with Borda rule, as illustrated by Figure 3. (Note that Figure 1(a) captures the case of $m=2$ for $r^{A}, r^{B}$, and $r^{P}$, too.) Whether such sensitivity is (un)desirable from an institutional perspective will depend on context and objectives. Higher sensitivity can give bigger incentives for political parties to campaign or private investment in voting stock. However, this needs to be weighed against other properties of a voting method such as consistency with respect to subgroup decisions (Young 1975), informational requirements, or complexity of strategic manipulation.

\section{Concluding remarks}

Equivalence of seemingly different distributions of seats, quotas, voting stock, etc. depends highly on whether decisions involve two, three, or more alternatives. Weight distributions such as $(6,5,2),(1,1,1)$, or $(48 \%, 24 \%, 28 \%)$ induce the same binary majority choices and Copeland decisions but lead to non-equivalent mappings from preferences to selected alternatives in other cases. The respective potential for weight differences to matter has been formalized and compared here. The investigated equivalences and their voting power implications (see Kurz et al. 2019) could be of interest not only for multicandidate voting in corporations, councils, or parliaments; e.g., acceptable polling error should be larger in the middle of an equivalence class 


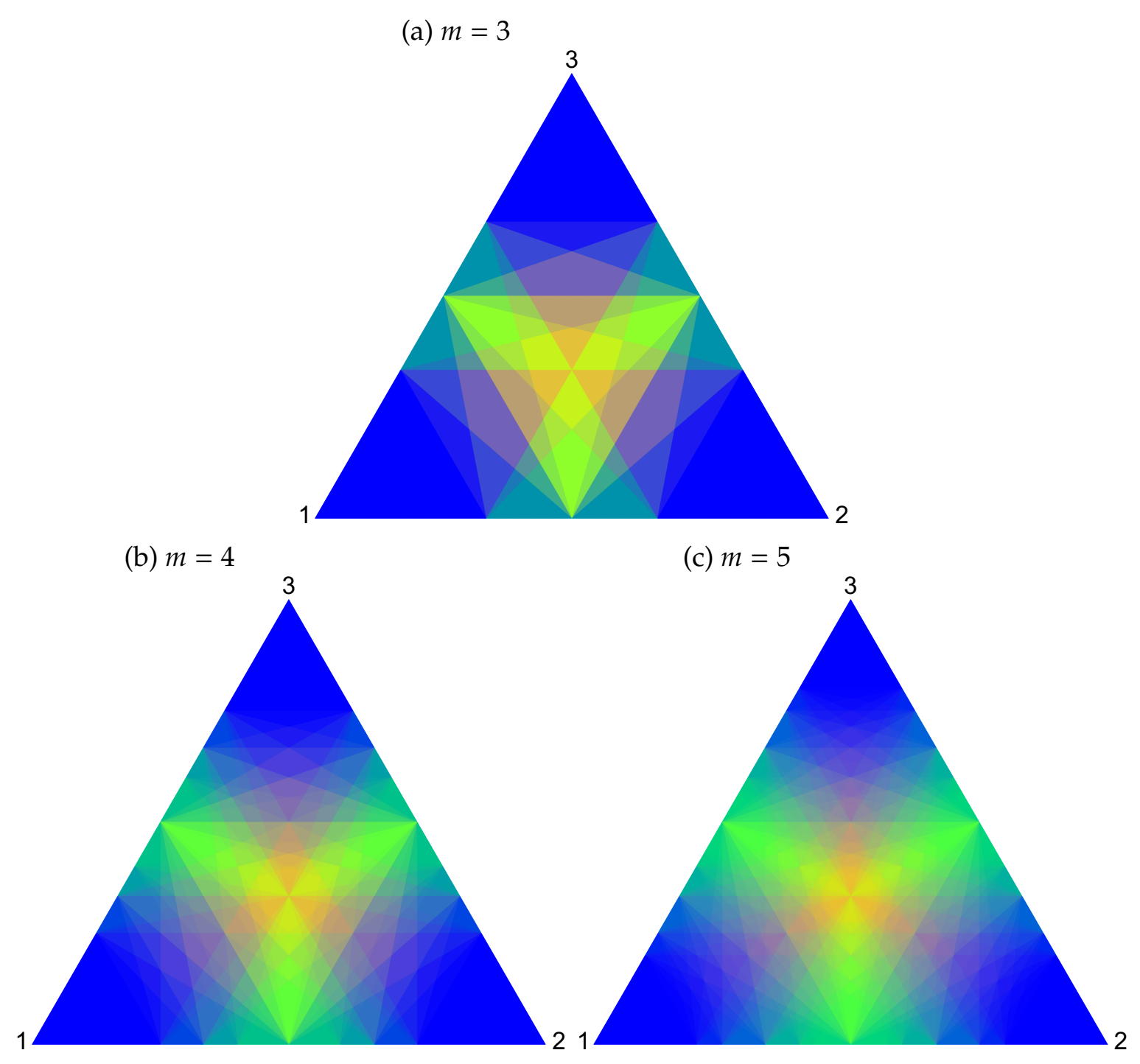

Figure 3: The 51, 505, and $\geq 2251$ Borda equivalence classes 
of an election rule than on its boundary.

For the IMF's Executive Board, we have checked that the 2016 reform of drawing rights can have consequences for who becomes the next IMF Managing Director. Election winners differ between reformed and unreformed weights for about $5 \%$ of all $6^{24}$ conceivable strict preference configurations over three shortlisted candidates. It matters for almost $15 \%$ of profiles whether Copeland or plurality rule is applied.

There is ample choice for extending above analysis since the list of single-winner voting procedures used by committees is long (see, e.g., Laslier 2012). We have tentatively considered scoring rules with arbitrary $\mathbf{s}=\left(1, s_{2}, 0\right) \in \mathbb{Q}^{3}$ for $n=m=3$, too. The numbers of distinct committees are M-shaped: they increase from 6 for plurality to more than 160 for $s_{2}=0.25$, fall to 51 Borda committees for $s_{2}=0.5$, increase again to at least 229 for $s_{2}=0.9$ and then drop to 5 antiplurality committees.

Implications of different weights are, obviously, just one aspect of preference aggregation by voting among others (cf. Nurmi 1987). Group decisions may often be purely binary affairs and the fast-growing dimensionality of collective choice makes it challenging to evaluate weighted voting for $m \geq 3$. Table 4 and Figures 13 document how non-trivial the links between weight and choice differences can be. They seem relevant enough, however, to be studied beyond the binary case.

\section{References}

Aleskerov, F. and E. Kurbanov (1999). Degree of manipulability of social choice procedures. In A. Alkan, C. D. Aliprantis, and N. C. Yannelis (Eds.), Current Trends in Economics, pp. 13-27. Berlin: Springer.

Amer, R., F. Carreras, and A. Magãna (1998). Extension of values to games with multiple alternatives. Annals of Operations Research 84(0), 63-78.

Bolger, E. M. (1983). The Banzhaf index for multicandidate presidential elections. SIAM Journal on Algebraic and Discrete Methods 4(4), 442-458.

Bouton, L. (2013). A theory of strategic voting in runoff elections. American Economic Review 103(4), 1248-1288.

Brams, S. J. and P. C. Fishburn (1996). Minimal winning coalitions in weighted-majority voting games. Social Choice and Welfare 13(4), 397-417.

Cheung, W.-S. and T.-W. Ng (2014). A three-dimensional voting system in Hong Kong. European Journal of Operational Research 236(1), 292-297. 
Chua, V. C. H., C. H. Ueng, and H. C. Huang (2002). A method for evaluating the behavior of power indices in weighted plurality games. Social Choice and Welfare 19(3), 665-680.

Felsenthal, D. S. and M. Machover (1997). Ternary voting games. International Journal of Game Theory 26(3), 335-351.

Felsenthal, D. S. and H. Nurmi (2017). Monotonicity Failures Afflicting Procedures for Electing a Single Candidate. Cham: Springer.

Freixas, J., M. Freixas, and S. Kurz (2017). On the characterization of weighted simple games. Theory and Decision 83(4), 469-498.

Freixas, J. and S. Kaniovski (2014). The minimum sum representation as an index of voting power. European Journal of Operational Research 233(3), 739-748.

Freixas, J. and W. S. Zwicker (2003). Weighted voting, abstention, and multiple levels of approval. Social Choice and Welfare 21(3), 399-431.

Henriet, D. (1985). The Copeland choice function: an axiomatic characterization. Social Choice and Welfare 2(1), 49-63.

Houy, N. and W. S. Zwicker (2014). The geometry of voting power: weighted voting and hyper-ellipsoids. Games and Economic Behavior 84, 7-16.

Hsiao, C.-R. and T. E. S. Raghavan (1993). Shapley value for multichoice cooperative games, I. Games and Economic Behavior 5(2), 240-256.

Krohn, I. and P. Sudhölter (1995). Directed and weighted majority games. Mathematical Methods of Operations Research 42(2), 189-216.

Kurihara, T. (2018). A simple characterization of the anti-plurality rule. Economics Letters 168, $110-111$.

Kurz, S. (2012a). On minimum sum representations for weighted voting games. Annals of Operations Research 196(1), 361-369.

Kurz, S. (2012b). On the inverse power index problem. Optimization 61(8), 989-1011.

Kurz, S., A. Mayer, and S. Napel (2019). Influence in weighted committees. Mimeo, University of Bayreuth.

Kurz, S. and S. Napel (2016). Dimension of the Lisbon voting rules in the EU Council: A challenge and new world record. Optimization Letters 10(6), 1245-1256.

Laruelle, A. and F. Valenciano (2012). Quaternary dichotomous voting rules. Social Choice and Welfare 38(3), 431-454.

Laslier, J.-F. (2012). And the loser is ...plurality voting. In D. S. Felsenthal and M. Machover (Eds.), Electoral Systems: Paradoxes, Assumptions, and Procedures, pp. 327-351. Berlin: Springer. 
Monroy, L. and F. R. Fernández (2009). A general model for voting systems with multiple alternatives. Applied Mathematics and Computation 215(4), 1537-1547.

Monroy, L. and F. R. Fernández (2011). The Shapley-Shubik index for multi-criteria simple games. European Journal of Operational Research 209(2), 122-128.

Moulin, H. (1988). Condorcet's principle implies the no show paradox. Journal of Economic Theory 45(1), 53-64.

Muroga, S. (1971). Threshold Logic and its Applications. New York, NY: Wiley.

Myerson, R. B. and R. J. Weber (1993). A theory of voting equilibria. American Political Science Review 87(1), 102-114.

Nurmi, H. (1987). Comparing Voting Systems. Dordrecht: Kluwer.

Saari, D. G. (1995). Basic Geometry of Voting. Berlin: Springer.

Saari, D. G. (2001). Chaotic Elections: A Mathematician Looks at Voting. Providence, RI: American Mathematical Society.

Taylor, A. D. and W. S. Zwicker (1999). Simple Games. Princeton, NJ: Princeton University Press.

Von Neumann, J. and O. Morgenstern (1953). Theory of Games and Economic Behavior (3rd ed.). Princeton, NJ: Princeton University Press.

Young, H. P. (1975). Social choice scoring functions. SIAM Journal on Applied Mathematics 28(4), 824-838. 


\section{Appendix A: Proofs}

\section{Proof of Proposition 3}

The claim is obvious for $n=1$. So consider $m \geq n+1$ for $n \geq 2$. Let $A=\left\{a_{1}, \ldots, a_{m}\right\}$ and $\mathbf{P}^{\mathbf{i}} \in \mathcal{P}(A)^{n}$ be any preference profile where the first $i$ players rank alternative $a_{1}$ last and the remaining $n-i$ players rank alternative $a_{2}$ last. Consider any $\overline{\mathbf{w}}_{\mathbf{k}}$ and $\overline{\mathbf{w}}_{\mathbf{l}}$ with $k<l$. Then $r^{A}\left|\overline{\mathbf{w}}_{\mathbf{k}}\left(\mathbf{P}^{\mathbf{k}}\right)=a_{2} \neq r^{A}\right| \overline{\mathbf{w}}_{\mathbf{l}}\left(\mathbf{P}^{\mathbf{k}}\right)=a_{3}$. So $\mathcal{E}_{\overline{\mathbf{w}}_{1}, m^{\prime}}^{r^{A}} \mathcal{E}_{\overline{\mathbf{w}}_{2}, m^{A}}^{r^{A}}, \ldots, \mathcal{E}_{\overline{\mathbf{w}}_{\mathbf{n}}, m}^{r^{A}}$ all differ.

Now assume $\mathbf{w} \in \mathbb{N}_{0}^{n} \backslash\{\mathbf{0}\}$ with $w_{1} \geq w_{2} \geq \ldots \geq w_{n}$ satisfies $\left(r^{A}, \mathbf{w}\right) \varkappa_{m}\left(r^{A}, \overline{\mathbf{w}}_{\mathbf{k}}\right)$ for all $k \in\{1, \ldots, n\}$. Let $l$ denote the index such that $w_{l}>0$ and $w_{l+1}=0$. Then both $r^{A} \mid \mathbf{w}(\mathbf{P})$ and $r^{A} \mid \overline{\mathbf{w}}_{\mathbf{l}}(\mathbf{P})$ equal the lexicographically minimal element in $Z^{l}(\mathbf{P}):=\left\{a \in A \mid \forall i \in\{1, \ldots, l\}: \exists a^{\prime} \in\right.$ $\left.A: a P_{i} a^{\prime}\right\}$, which collects all alternatives not ranked last by any of the players who have positive weight. These coincide for $\mathbf{w}$ and $\overline{\mathbf{w}}_{1}$; and $Z^{l}(\mathbf{P})$ is non-empty because $m \geq n+1$. This holds for arbitrary $\mathbf{P} \in \mathcal{P}(A)^{n}$. Hence $r^{A}\left|\mathbf{w} \equiv r^{A}\right| \overline{\mathbf{w}}_{1}$, contradicting the assumption that $\left(r^{A}, \mathbf{w}\right) \varkappa_{m}\left(r^{A}, \overline{\mathbf{w}}_{\mathbf{k}}\right)$ for all $k \in\{1, \ldots, n\}$. Consequently, $\mathcal{E}_{\overline{\mathbf{w}}_{1}, m^{\prime}}^{r^{A}} \mathcal{E}_{\overline{\mathbf{w}}_{2}, m^{A}}^{r^{A}}, \ldots, \mathcal{E}_{\overline{\mathbf{w}}_{\mathbf{n}}, m}^{r^{A}}$ are all antiplurality classes that exist for $m \geq n+1$ (plus the degenerate $\mathcal{E}_{0, m}$ ).

\section{Proof of Proposition 4}

Let $k>j$ for otherwise arbitrary $j, k \in\{1, \ldots, m\}$ and consider any profile $\mathbf{P} \in \mathcal{P}(A)^{n}$ such that $a_{2} P_{1} a_{1} P_{1} a_{3} P_{1} a_{4} \ldots a_{m}$ and $a_{1} P_{2} a_{3} P_{2} a_{4} \ldots a_{k} P_{2} a_{2} P_{2} a_{k+1} P_{2} a_{k+2} \ldots a_{m}$. The Borda score $j \cdot(m-2)+(m-1)$ of $a_{1}$ under $\tilde{\mathbf{w}}_{\mathbf{j}}$ is at least as big as the corresponding score $j \cdot(m-1)+(m-k)$ of $a_{2}$. Since scores of $a_{3}, \ldots, a_{m}$ are all strictly smaller than that of $a_{1}$, we have $r^{B} \mid \tilde{\mathbf{w}}_{\mathbf{j}}(P)=a_{1}$. With $\tilde{\mathbf{w}}_{\mathbf{k}}$, by contrast, $a_{1}{ }^{\prime}$ s weighted score $k \cdot(m-2)+(m-1)$ is strictly smaller than $a_{2}{ }^{\prime} \mathbf{s}$ corresponding score $k \cdot(m-1)+(m-k)$. Scores of $a_{3}, \ldots, a_{m}$ remain smaller than $a_{1}$ 's. So $r^{B} \mid \tilde{\mathbf{w}}_{\mathbf{k}}(P)=a_{2}$. Hence $\left(r^{B}, \tilde{\mathbf{w}}_{\mathbf{j}}\right) \varkappa_{m}\left(r^{B}, \tilde{\mathbf{w}}_{\mathbf{k}}\right)$.

\section{Proof of Proposition 5}

For a given set $A=\left\{a_{1}, \ldots, a_{m}\right\}$ and any subset $A^{\prime} \subseteq A$ that preserves the order of the alternatives, denote the projection of preference profile $\mathbf{P} \in \mathcal{P}(A)^{n}$ to $A^{\prime}$ by $\mathbf{P} \downarrow_{A^{\prime}}$ with $a_{k} P_{i} \downarrow_{A^{\prime}}$ $a_{l}: \Leftrightarrow\left[a_{k} P_{i} a_{l}\right.$ and $\left.a_{k}, a_{l} \in A^{\prime}\right]$. Conversely, if $A^{\prime} \supseteq A$ is a superset of $A$ with $A^{\prime} \backslash A=$ $\left\{a_{m+1}, \ldots, a_{m^{\prime}}\right\}$ define the lifting $\mathbf{P} \uparrow A^{A^{\prime}}$ of $\mathbf{P} \in \mathcal{P}(A)^{n}$ to $A^{\prime}$ by appending alternatives $a_{m+1}, \ldots, a_{m^{\prime}}$ to each ordering $P_{i}$ below the lowest-ranked alternative from $A$.

Now consider $m>2$ and $\mathbf{w}, \mathbf{w}^{\prime} \in \mathbb{N}_{0}^{n}$ such that $\left(r^{C}, \mathbf{w}\right){ }_{m}\left(r^{C}, \mathbf{w}^{\prime}\right)$, i.e., ${ }^{C}\left|\mathbf{w}(\mathbf{P}) \neq r^{C}\right| \mathbf{w}^{\prime}(\mathbf{P})$ for some $\mathbf{P} \in \mathcal{P}(A)^{n}$. The $\mathbf{w}$ and $\mathbf{w}^{\prime}$-weighted versions of the majority relation must differ at 
P. W.l.o.g. let weak victory of $a_{k}$ over $a_{l}$ for $\mathbf{w}$ turn into a strict victory of $a_{l}$ over $a_{k}$ for $\mathbf{w}^{\prime}$ :

$$
\sum_{i: a_{k} P_{i} a_{l}} w_{i} \geq \sum_{j: a_{l} P_{j} a_{k}} w_{j} \text { and } \sum_{i: a_{k} P_{i} a_{l}} w_{i}^{\prime}<\sum_{j: a_{l} P_{j} a_{k}} w_{j}^{\prime} .
$$

Then take $A^{\prime}=\left\{a_{k}, a_{l}\right\} \subset A$ where $\left|A^{\prime}\right|=2$ and projection $\mathbf{P} \downarrow_{A^{\prime}} \cdot(6)$ implies

$$
\sum_{i: a_{k} P_{i} \downarrow_{A^{\prime}} a_{l}} w_{i} \geq \sum_{j: a_{l} P_{j} \downarrow_{A^{\prime}} a_{k}} w_{j} \text { and } \sum_{i: a_{k} P_{i} \downarrow_{A^{\prime}} a_{l}} w_{i}^{\prime}<\sum_{j: a_{l} P_{j} \downarrow_{A^{\prime}} a_{k}} w_{j}^{\prime} .
$$

If both inequalities are strict or $k<l$ then $r^{C}\left|\mathbf{w}\left(\mathbf{P} \downarrow_{A^{\prime}}\right)=a_{k} \neq r^{C}\right| \mathbf{w}^{\prime}\left(\mathbf{P} \downarrow_{A^{\prime}}\right)=a_{l}$ and hence $\left(r^{C}, \mathbf{w}\right) \nsim_{2}\left(r^{C}, \mathbf{w}^{\prime}\right)$. If not, $a_{l}$ wins also for $\mathbf{w}$ by lexicographic tie breaking but we can consider profile $\mathbf{P}^{\prime} \in \mathcal{P}\left(A^{\prime}\right)^{n}$ with $a_{l} P_{i}^{\prime} a_{k} \Leftrightarrow a_{k} P_{i} \downarrow_{A^{\prime}} a_{l}$ for all $i \in N$. Then $r^{C}\left|\mathbf{w}\left(\mathbf{P}^{\prime}\right)=a_{l} \neq r^{C}\right| \mathbf{w}^{\prime}\left(\mathbf{P}^{\prime}\right)=a_{k}$ and $\left(r^{C}, \mathbf{w}\right) \star_{2}\left(r^{C}, \mathbf{w}^{\prime}\right)$.

Conversely take $A=\left\{a_{1}, a_{2}\right\}$ and $\mathbf{w}, \mathbf{w}^{\prime} \in \mathbb{N}_{0}^{n}$ such that $\left(r^{C}, \mathbf{w}\right) \varkappa_{2}\left(r^{C}, \mathbf{w}^{\prime}\right)$ and $r^{C} \mid \mathbf{w}(\mathbf{P})=$ $a_{1} \neq r^{C} \mid \mathbf{w}^{\prime}(\mathbf{P})=a_{2}$ for some $\mathbf{P} \in \mathcal{P}(A)^{n}$. Then

$$
\sum_{i: a_{1} P_{i} a_{2}} w_{i} \geq \sum_{j: a_{2} P_{j} a_{1}} w_{j} \text { and } \sum_{i: a_{1} P_{i} a_{2}} w_{i}^{\prime}<\sum_{j: a_{2} P_{j} a_{1}} w_{j}^{\prime} .
$$

Consider $A^{\prime}=\left\{a_{1}, a_{2}, \ldots, a_{m}\right\} \supset A$ where $\left|A^{\prime}\right|=m$ and lifting $\mathbf{P} \uparrow^{A^{\prime}} .(8)$ implies

$$
\sum_{i: a_{1} P_{i} \uparrow^{A^{\prime}} a_{2}} w_{i} \geq \sum_{j: a_{2} P_{j} \uparrow^{A^{\prime}} a_{1}} w_{j} \text { and } \sum_{i: a_{1} P_{i} \uparrow^{A^{\prime} a_{2}}} w_{i}^{\prime}<\sum_{j: a_{2} P_{j} \uparrow^{A^{\prime}} a_{1}} w_{j}^{\prime}
$$

and alternatives $a_{3}, \ldots, a_{m}$ lose all weighted majority comparisons against $a_{1}$ and $a_{2}$ by construction of $\mathbf{P} \uparrow^{A^{\prime}}$. So $r^{C}\left|\mathbf{w}\left(\mathbf{P} \uparrow^{A^{\prime}}\right)=a_{1} \neq r^{C}\right| \mathbf{w}^{\prime}\left(\mathbf{P} \uparrow^{A^{\prime}}\right)=a_{2}$. Hence $\left(r^{C}, \mathbf{w}\right) \nsim_{m}\left(r^{C}, \mathbf{w}^{\prime}\right)$. In summary, $\left(r^{C}, \mathbf{w}\right) \nsim_{2}\left(r^{C}, \mathbf{w}^{\prime}\right) \Leftrightarrow\left(r^{C}, \mathbf{w}\right) \nsim_{m}\left(r^{C}, \mathbf{w}^{\prime}\right)$ and, a fortiori, $\left(r^{C}, \mathbf{w}\right) \sim_{2}\left(r^{C}, \mathbf{w}^{\prime}\right) \Leftrightarrow$ $\left(r^{C}, \mathbf{w}\right) \sim_{m}\left(r^{C}, \mathbf{w}^{\prime}\right)$.

\section{Proof of Proposition 6}

Let $m>n$. Consider $A=\left\{a_{1}, \ldots, a_{m}\right\}$ and any $\mathbf{w}, \mathbf{w}^{\prime} \in \mathbb{N}_{0}^{n}$ such that $\left(r^{P}, \mathbf{w}\right) \varkappa_{m}\left(r^{P}, \mathbf{w}^{\prime}\right)$. So there exists $\mathbf{P} \in \mathcal{P}(A)^{n}$ with $r^{P}\left|\mathbf{w}(\mathbf{P})=a_{k} \neq r^{P}\right| \mathbf{w}^{\prime}(\mathbf{P})=a_{l}$. For this $\mathbf{P}$ let $\hat{A}:=\left\{a \mid \exists i \in N: \forall a^{\prime} \neq a: a P_{i} a^{\prime}\right\}$ denote the set of all alternatives that are top-ranked by some voter. (Obviously, $a_{k}, a_{l} \in \hat{A}$.) Now define $A^{\prime} \subset A$ as the union of $\hat{A}$ and some arbitrary elements of $A \backslash \hat{A}$ such that $\left|A^{\prime}\right|=n$. By construction, each $a \in A^{\prime}$ has the same weighted number of top positions for projection $\mathbf{P} \downarrow_{A^{\prime}}$ as it had for $\mathbf{P}$. So $r^{P}\left|\mathbf{w}\left(\mathbf{P} \downarrow_{A^{\prime}}\right)=a_{k} \neq r^{P}\right| \mathbf{w}^{\prime}\left(\mathbf{P} \downarrow_{A^{\prime}}\right)=a_{l}$. Hence $\left(r^{P}, \mathbf{w}\right) \nsim_{n}\left(r^{P}, \mathbf{w}^{\prime}\right)$.

Analogously, consider $A=\left\{a_{1}, \ldots, a_{n}\right\}$ and $\mathbf{w}, \mathbf{w}^{\prime} \in \mathbb{N}_{0}^{n}$ such that $\left(r^{P}, \mathbf{w}\right) \varkappa_{n}\left(r^{P}, \mathbf{w}^{\prime}\right)$. A profile $\mathbf{P} \in \mathcal{P}(A)^{n}$ with $r^{P}\left|\mathbf{w}(\mathbf{P})=a_{k} \neq r^{P}\right| \mathbf{w}^{\prime}(\mathbf{P})=a_{l}$ can then be lifted to $A^{\prime}=A \cup\left\{a_{n+1}, \ldots, a_{m}\right\}$. By construction, $r^{P}\left|\mathbf{w}\left(\mathbf{P} \uparrow A^{\prime}\right)=a_{k} \neq r^{P}\right| \mathbf{w}^{\prime}\left(\mathbf{P} \uparrow^{A^{\prime}}\right)=a_{l}$. Hence $\left(r^{P}, \mathbf{w}\right) x_{m}\left(r^{P}, \mathbf{w}^{\prime}\right)$. Overall, we can conclude $\left(r^{P}, \mathbf{w}\right) \sim_{m}\left(r^{P}, \mathbf{w}^{\prime}\right) \Leftrightarrow\left(r^{P}, \mathbf{w}\right) \sim_{n}\left(r^{P}, \mathbf{w}^{\prime}\right)$. 


\section{Appendix B: Minimal representations of committees}

\begin{tabular}{||c|cc|cc|cc|cc||}
\hline \hline$n, m$ & \multicolumn{6}{|c||}{ Minimal $\overline{\mathbf{w}}$ for all antiplurality classes $\mathcal{E}_{\overline{\mathbf{w}}, m}^{r^{4}}$} \\
\hline \hline 3,3 & 1. & $(1,0,0)$ & 3. & $(1,1,1)$ & 5. & $(2,2,1)$ & & \\
& 2. & $(1,1,0)$ & 4. & $(2,1,1)$ & & & & \\
\hline \hline $3, m \geq 4$ & 1. & $(1,0,0)$ & 2. & $(1,1,0)$ & 3. & $(1,1,1)$ & & \\
\hline \hline 4,3 & 1. & $(1,0,0,0)$ & 6. & $(2,1,1,1)$ & 11. & $(3,2,2,1)$ & 16. & $(4,3,2,2)$ \\
& 2. & $(1,1,0,0)$ & 7. & $(2,2,1,0)$ & 12. & $(3,3,1,1)$ & 17. & $(4,4,2,1)$ \\
& 3. & $(1,1,1,0)$ & 8. & $(2,2,1,1)$ & 13. & $(3,3,2,1)$ & 18. & $(4,4,3,2)$ \\
& 4. & $(1,1,1,1)$ & 9. & $(2,2,2,1)$ & 14. & $(3,3,2,2)$ & 19. & $(5,4,3,2)$ \\
& 5. & $(2,1,1,0)$ & 10. & $(3,2,1,1)$ & 15. & $(4,3,2,1)$ & & \\
\hline \hline 4,4 & 1. & $(1,0,0,0)$ & 3. & $(1,1,1,0)$ & 5. & $(2,1,1,1)$ & 7. & $(2,2,2,1)$ \\
& 2. & $(1,1,0,0)$ & 4. & $(1,1,1,1)$ & 6. & $(2,2,1,1)$ & & \\
\hline \hline $4, m \geq 5$ & 1. & $(1,0,0,0)$ & 2. & $(1,1,0,0)$ & 3. & $(1,1,1,0)$ & 4. & $(1,1,1,1)$ \\
\hline \hline
\end{tabular}

Table B-1: Minimal representations of different antiplurality committees

\begin{tabular}{||c|cc|cc|cc|cc||}
\hline \hline$n, m$ & \multicolumn{6}{|c||}{ Minimal $\overline{\mathbf{w}}$ for all Borda classes $\mathcal{E}_{\overline{\mathbf{w}}, 3}^{r^{B}}$} \\
\hline \hline 3,3 & 1. & $(1,0,0)$ & 14. & $(3,3,2)$ & 27. & $(5,4,3)$ & 40. & $(8,6,3)$ \\
& 2. & $(1,1,0)$ & 15. & $(4,3,1)$ & 28. & $(7,4,1)$ & 41. & $(9,6,2)$ \\
& 3. & $(1,1,1)$ & 16. & $(5,2,1)$ & 29. & $(6,5,2)$ & 42. & $(8,7,3)$ \\
& 4. & $(2,1,0)$ & 17. & $(4,3,2)$ & 30. & $(7,5,1)$ & 43. & $(8,6,5)$ \\
& 5. & $(2,1,1)$ & 18. & $(5,2,2)$ & 31. & $(6,5,3)$ & 44. & $(10,7,2)$ \\
& 6. & $(2,2,1)$ & 19. & $(5,3,1)$ & 32. & $(7,5,2)$ & 45. & $(11,7,2)$ \\
& 7. & $(3,1,1)$ & 20. & $(4,3,3)$ & 33. & $(8,5,1)$ & 46. & $(9,7,5)$ \\
& 8. & $(3,2,0)$ & 21. & $(5,4,1)$ & 34. & $(6,5,4)$ & 47. & $(10,8,3)$ \\
& 9. & $(3,2,1)$ & 22. & $(6,3,1)$ & 35. & $(7,5,3)$ & 48. & $(11,8,2)$ \\
& 10. & $(4,1,1)$ & 23. & $(5,3,3)$ & 36. & $(7,6,2)$ & 49. & $(11,9,3)$ \\
& 11. & $(3,2,2)$ & 24. & $(5,4,2)$ & 37. & $(8,5,2)$ & 50. & $(13,8,2)$ \\
& 12. & $(3,3,1)$ & 25. & $(6,4,1)$ & 38. & $(7,5,4)$ & 51. & $(12,9,7)$ \\
& 13. & $(4,2,1)$ & 26. & $(7,2,2)$ & 39. & $(7,6,4)$ & & \\
\hline \hline
\end{tabular}

Table B-2: Minimal representations of different Borda committees 


\begin{tabular}{|c|c|c|c|c|c|}
\hline$n$ & \multicolumn{5}{|c|}{$\begin{array}{l}\text { Minimal } \overline{\mathbf{w}} \text { for all Copeland classes } \mathcal{E}_{\overline{\mathbf{w}}, m}^{r^{C}} \\
\text { and for all classes } \mathcal{E}_{\overline{\mathbf{w}}, 2}^{r} \text { when } r \in\left\{r^{A}, r^{B}, r^{P}\right\} \\
\text { and for all weighted voting games }[q ; \mathbf{w}] \text { with } q=0.5 \sum w_{i}\end{array}$} \\
\hline 3 & $(1,0,0)$ & $(1,1,0)$ & 3. $\quad(1,1,1)$ & & $(211)$ \\
\hline 4 & $\begin{array}{l}(1,0,0,0) \\
(1,1,0,0) \\
(1,1,1,0)\end{array}$ & $\begin{array}{ll}4 . & (1,1,1,1) \\
5 . & (2,1,1,0) \\
6 . & (2,1,1,1)\end{array}$ & $\begin{array}{ll}7 . & (2,2,1,1) \\
8 . & (3,1,1,1) \\
9 . & (3,2,2,1)\end{array}$ & & \\
\hline 5 & $\begin{array}{ll}1 \text { 1. } & (1,0,0,0,0) \\
\text { 2. } & (1,1,0,0,0) \\
\text { 3. } & (1,1,1,0,0) \\
\text { 4. } & (1,1,1,1,0) \\
\text { 5. } & (2,1,1,0,0) \\
\text { 6. } & (1,1,1,1,1) \\
\text { 7. } & (2,1,1,1,0)\end{array}$ & $\begin{aligned} 8 . & (2,1,1,1,1) \\
9 . & (2,2,1,1,0) \\
10 . & (3,1,1,1,0) \\
11 . & (2,2,1,1,1) \\
12 . & (3,1,1,1,1) \\
13 . & (2,2,2,1,1) \\
14 . & (3,2,1,1,1)\end{aligned}$ & $\begin{array}{ll}15 . & (3,2,2,1,0) \\
16 . & (4,1,1,1,1) \\
17 . & (3,2,2,1,1) \\
18 . & (3,2,2,2,1) \\
19 . & (3,3,2,1,1) \\
20 . & (4,2,2,1,1) \\
21 . & (3,3,2,2,2)\end{array}$ & $\begin{array}{l}22 . \\
23 . \\
24 . \\
25 . \\
26 . \\
27 .\end{array}$ & $\begin{array}{l}(4,3,2,2,1) \\
(4,3,3,1,1) \\
(5,2,2,2,1) \\
(4,3,3,2,2) \\
(5,3,3,2,1) \\
(5,4,3,2,2)\end{array}$ \\
\hline & & ee $n$ & & & \\
\hline
\end{tabular}




\begin{tabular}{|c|c|c|c|c|c|c|c|c|}
\hline 5 & \multicolumn{8}{|c|}{... see previous page } \\
\hline \multirow[t]{35}{*}{6} & 1. & $(1,0,0,0,0,0)$ & 36 & $\overline{(3,2,2,2,2,1)}$ & & $(5,4,3,2,1,1)$ & 106 & $(5,5,4,3,3,2)$ \\
\hline & & $(1,1,0,0,0,0)$ & 37 & $(3,3,2,2,1,1)$ & 72 & $(5,4,3,2,2,0)$ & 107 & $(6,4,4,3,3,2)$ \\
\hline & 3. & $(1,1,1,0,0,0)$ & 38 & $(3,3,2,2,2,0)$ & 73 & $(5,4,4,1,1,1)$ & 108 & $(6,5,4,3,2,2)$ \\
\hline & 4. & $(1,1,1,1,0,0)$ & 39 & $(3,3,3,1,1,1)$ & 74 & $(6,3,2,2,2,1)$ & 109 & $(6,5,4,3,3,1)$ \\
\hline & 5. & $(2,1,1,0,0,0)$ & 40 & $(4,2,2,2,1,1)$ & 75 & $(6,3,3,2,1,1)$ & 110 & $(6,5,5,2,2,2)$ \\
\hline & & $(1,1,1,1,1,0)$ & 41 & $(4,3,2,1,1,1)$ & 76 & $(7,2,2,2,2,1)$ & 111 & $(7,4,4,3,2,2)$ \\
\hline & 7. & $(2,1,1,1,0,0)$ & 42 & $(4,3,2,2,1,0)$ & 77 & $(5,4,3,2,2,1)$ & 112 & $(7,5,3,3,2,2)$ \\
\hline & 8. & $(1,1,1,1,1,1)$ & 43 & $(4,3,3,1,1,0)$ & 78 & $(4,4,3,3,2,2)$ & 113 & $(7,5,4,3,2,1)$ \\
\hline & & $(2,1,1,1,1,0)$ & 44 & $(5,2,2,1,1,1)$ & 79 & $(4,4,3,3,3,1)$ & 114 & $(7,5,5,2,2,1)$ \\
\hline & 10 . & $(2,2,1,1,0,0)$ & 45 & $(5,2,2,2,1,0)$ & 80 & $(5,3,3,3,2,2)$ & 115 & $(8,4,3,3,2,2)$ \\
\hline & & $(3,1,1,1,0,0)$ & 46 & $(3,3,2,2,2,1)$ & 81 & $(5,4,3,2,2,2)$ & 116 & $(6,5,4,4,3,2)$ \\
\hline & & $(2,1,1,1,1,1)$ & 47 & $(4,3,2,2,1,1)$ & 82 & $(5,4,3,3,2,1)$ & 117 & $(6,5,5,3,3,2)$ \\
\hline & & $(2,2,1,1,1,0)$ & 48 & $(4,3,3,1,1,1)$ & 83 & $(5,4,4,2,2,1)$ & 118 & $(7,5,4,3,3,2)$ \\
\hline & 14 . & $(3,1,1,1,1,0)$ & 49 & $(5,2,2,2,1,1)$ & 84 & $(5,5,3,2,2,1)$ & 119 & $(7,5,4,4,2,2)$ \\
\hline & 15 . & $(2,2,1,1,1,1)$ & 50 & $(3,3,2,2,2,2)$ & 85 & $(6,3,3,2,2,2)$ & 120 & $(7,5,5,3,3,1)$ \\
\hline & & $(2,2,2,1,1,0)$ & 51 & $(3,3,3,2,2,1)$ & 86 & $(6,4,3,2,2,1)$ & 121 & $(7,6,4,3,2,2)$ \\
\hline & & $(3,1,1,1,1,1)$ & 52 & $(4,3,2,2,2,1)$ & 87 & $(6,4,3,3,1,1)$ & 122 & $(7,6,4,3,3,1)$ \\
\hline & 18. & $(3,2,1,1,1,0)$ & 53 & $(4,3,3,2,1,1)$ & 88 & $(6,4,4,2,1,1)$ & 123 & $(7,6,5,2,2,2)$ \\
\hline & & $(3,2,2,1,0,0)$ & 54 & $(4,3,3,2,2,0)$ & 89 & $(7,3,3,2,2,1)$ & 124 & $(8,5,4,3,2,2)$ \\
\hline & 20. & $(4,1,1,1,1,0)$ & 55 & $(4,4,2,2,1,1)$ & 90 & $(7,3,3,3,1,1)$ & 125 & $(8,5,5,3,2,1)$ \\
\hline & 21 . & $(2,2,2,1,1,1)$ & 56 & $(4,4,3,1,1,1)$ & 91 & $(5,4,3,3,3,2)$ & 126 & $(9,4,4,3,2,2)$ \\
\hline & 22 . & $(3,2,1,1,1,1)$ & 57 & $(5,2,2,2,2,1)$ & 92 & $(5,4,4,3,2,2)$ & 127 & $(7,5,5,4,3,2)$ \\
\hline & 23. & $(3,2,2,1,1,0)$ & 58 & $(5,3,2,2,1,1)$ & 93 & $(5,4,4,3,3,1)$ & 128 & $(7,6,5,3,3,2)$ \\
\hline & 24 & $(4,1,1,1,1,1)$ & 59 & $(5,3,3,1,1,1)$ & 94 & $(5,5,3,3,3,1)$ & 129 & $(8,5,5,4,2,2)$ \\
\hline & 25. & $(2,2,2,2,1,1)$ & 60 & $(5,3,3,2,1,0)$ & 95 & $(5,5,4,2,2,2)$ & 130 & $(8,6,4,3,3,2)$ \\
\hline & & $(3,2,2,1,1,1)$ & 61 & $(6,2,2,2,1,1)$ & 96 & $(6,4,3,3,2,2)$ & 131 & $(8,6,5,3,3,1)$ \\
\hline & & $(3,2,2,2,1,0)$ & 62 & $(4,3,3,2,2,1)$ & 97 & $(6,4,4,3,2,1)$ & 132 & $(9,5,5,3,2,2)$ \\
\hline & 28 . & $(3,3,1,1,1,1)$ & 63 & $(5,3,3,2,1,1)$ & 98 & $(6,5,3,2,2,2)$ & 133 & $(7,6,5,4,4,2)$ \\
\hline & 29. & $(3,3,2,1,1,0)$ & 64 & $(4,3,3,2,2,2)$ & 99 & $(6,5,3,3,2,1)$ & 134 & $(8,6,5,4,3,2)$ \\
\hline & 30. & $(4,2,1,1,1,1)$ & 65 & $(4,3,3,3,2,1)$ & 100 & $(6,5,4,2,2,1)$ & 135 & $(8,7,5,3,3,2)$ \\
\hline & & $(4,2,2,1,1,0)$ & 66 & $(4,4,3,2,2,1)$ & 101 & $(7,3,3,3,2,2)$ & 136 & $(9,6,5,4,2,2)$ \\
\hline & & $(5,1,1,1,1,1)$ & 67 & $(5,3,2,2,2,2)$ & 102 & $(7,4,3,2,2,2)$ & 137 & $(9,7,5,4,3,2)$ \\
\hline & 33. & $(3,2,2,2,1,1)$ & 68 & $(5,3,3,2,2,1)$ & 103 & $(7,4,4,2,2,1)$ & 138 & $(9,7,6,4,4,2)$ \\
\hline & & $(3,3,2,1,1,1)$ & 69 & $(5,3,3,3,1,1)$ & 104 & $(7,4,4,3,1,1)$ & & \\
\hline & & $(4,2,2,1,1,1)$ & 70 & $(5,4,2,2,2,1)$ & 105 & $(8,3,3,3,2,1)$ & & \\
\hline
\end{tabular}

Table B-3: Minimal representation of different Copeland committees for $m \geq 2$, and of different antiplurality, Borda and plurality committees for $m=2$, and of different weighted voting games with a simple majority 


\begin{tabular}{|c|cc|cc|cc|cc||}
\hline \hline$n, m$ & \multicolumn{8}{|c||}{ Minimal $\overline{\mathbf{w}}$ for all plurality classes $\mathcal{E}_{\overline{\mathbf{w}}, m}^{r^{p}}$} \\
\hline \hline $3, m \geq 3$ & 1. & $(1,0,0)$ & 3. & $(1,1,1)$ & 5. & $(2,2,1)$ & & \\
& 2. & $(1,1,0)$ & 4. & $(2,1,1)$ & 6. & $(3,2,2)$ & & \\
\hline \hline 4,3 & 1. & $(1,0,0,0)$ & 10. & $(2,2,2,1)$ & 19. & $(4,3,2,1)$ & 28. & $(5,4,3,1)$ \\
& 2. & $(1,1,0,0)$ & 11. & $(3,2,1,1)$ & 20. & $(4,3,2,2)$ & 29. & $(5,4,3,2)$ \\
& 3. & $(1,1,1,0)$ & 12. & $(3,2,2,0)$ & 21. & $(4,3,3,1)$ & 30. & $(6,4,3,2)$ \\
& 4. & $(1,1,1,1)$ & 13. & $(3,2,2,1)$ & 22. & $(4,4,2,1)$ & 31. & $(6,5,3,2)$ \\
& 5. & $(2,1,1,0)$ & 14. & $(3,3,1,1)$ & 23. & $(5,2,2,2)$ & 32. & $(6,5,4,2)$ \\
& 6. & $(2,1,1,1)$ & 15. & $(3,2,2,2)$ & 24. & $(4,3,3,2)$ & 33. & $(7,4,4,2)$ \\
& 7. & $(2,2,1,0)$ & 16. & $(3,3,2,1)$ & 25. & $(5,3,3,1)$ & 34. & $(7,6,4,2)$ \\
& 8. & $(2,2,1,1)$ & 17. & $(4,2,2,1)$ & 26. & $(5,3,3,2)$ & & \\
& 9. & $(3,1,1,1)$ & 18. & $(3,3,2,2)$ & 27. & $(5,4,2,2)$ & & \\
\hline \hline $4, m \geq 4$ & 1. & $(1,0,0,0)$ & 10. & $(2,2,2,1)$ & 19. & $(4,3,2,1)$ & 28. & $(5,4,2,2)$ \\
& 2. & $(1,1,0,0)$ & 11. & $(3,2,1,1)$ & 20. & $(4,3,2,2)$ & 29. & $(5,4,3,1)$ \\
& 3. & $(1,1,1,0)$ & 12. & $(3,2,2,0)$ & 21. & $(4,3,3,1)$ & 30. & $(5,4,3,2)$ \\
& 4. & $(1,1,1,1)$ & 13. & $(3,2,2,1)$ & 22. & $(4,4,2,1)$ & 31. & $(5,4,4,2)$ \\
& 5. & $(2,1,1,0)$ & 14. & $(3,3,1,1)$ & 23. & $(5,2,2,2)$ & 32. & $(6,4,3,2)$ \\
& 6. & $(2,1,1,1)$ & 15. & $(3,2,2,2)$ & 24. & $(4,3,3,2)$ & 33. & $(6,5,3,2)$ \\
& 7. & $(2,2,1,0)$ & 16. & $(3,3,2,1)$ & 25. & $(5,3,3,1)$ & 34. & $(6,5,4,2)$ \\
& 8. & $(2,2,1,1)$ & 17. & $(4,2,2,1)$ & 26. & $(4,4,3,2)$ & 35. & $(7,4,4,2)$ \\
& 9. & $(3,1,1,1)$ & 18. & $(3,3,2,2)$ & 27. & $(5,3,3,2)$ & 36. & $(7,6,4,2)$ \\
\hline \hline
\end{tabular}

Table B-4: Minimal representations of different plurality committees 